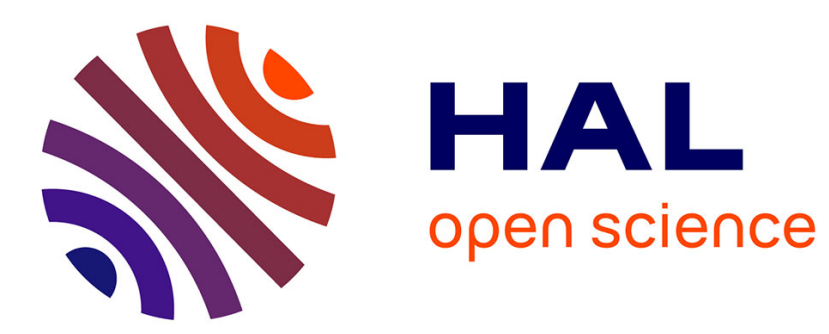

\title{
Piecewise Affine State Feedback Controller for Lane Departure Avoidance
}

Andre Benine-Neto, Said Mammar

\section{To cite this version:}

Andre Benine-Neto, Said Mammar. Piecewise Affine State Feedback Controller for Lane Departure Avoidance. IEEE Intelligent Vehicles Symposium (IV 2011), Jun 2011, Baden-Baden, Germany. pp.839-844, 10.1109/IVS.2011.5940496 . hal-00654102

\section{HAL Id: hal-00654102 \\ https://hal.science/hal-00654102}

Submitted on 5 Jul 2021

HAL is a multi-disciplinary open access archive for the deposit and dissemination of scientific research documents, whether they are published or not. The documents may come from teaching and research institutions in France or abroad, or from public or private research centers.
L'archive ouverte pluridisciplinaire HAL, est destinée au dépôt et à la diffusion de documents scientifiques de niveau recherche, publiés ou non, émanant des établissements d'enseignement et de recherche français ou étrangers, des laboratoires publics ou privés. 


\title{
Piecewise Affine State Feedback Controller for Lane Departure Avoidance
}

\author{
André Benine-Neto ${ }^{1}$, Saïd Mammar ${ }^{2}$
}

\begin{abstract}
This paper presents the design and simulation tests of a lane keeping assistance system for passenger vehicles based on a piecewise affine state feedback controller. The design of the proposed lane keeping system takes into account the entire domain of lateral tire forces through piecewise affine approximations of the tire forces nonlinear behavior. The computation of the control law is casted as Bilinear Matrix Inequalities optimization procedure which is solved using the V-K-method to find a piecewise quadratic Lyapunov function and the state feedback gain. Simulations show the improved performance of the controller on degraded road adhesion conditions.
\end{abstract}

\section{INTRODUCTION}

Lane departure represents a significant fraction of road accidents and in many cases it is due to the driver fatigue or inattentiveness, therefore research on vehicle lateral control has been gained importance over the last 40 years, either for full or partial automation of driving tasks, in order to increase safety by reducing accidents and drivers workload. An overview of the recent research activity is provided in [18]. It can be noted that earlier work was devoted to autonomous vehicles in highway scenarios in which the experienced lateral accelerations are generally low [7], which has also been considered in more recent works for the development of assistance systems which replace the driver during the lane departure avoidance manoeuvres [12]. Nevertheless, in order to provide more safety to the drivers, these Lane Keeping Assistance Systems (LKAS) must be able to operate in the complete domain of tire forces, including the nonlinear behavior [9], as many accidents occur due to the vehicle loss of control when the tire forces are saturated.

Vehicle lateral control in saturated tire force conditions has been proposed for autonomous lane keeping system in [6] and in [11] using a parallel distributed control structure developed on a Takagi-Sugeno model.

Piecewise Affine (PWA) systems [19] provide another possibility to model the nonlinearities. It has been employed in [4] for the design of traction controller and in [3] for a yaw rate tracking system both using a model predictive control strategy. PWA approximation of the tire forces has also been used in [17] and [2] to develop an active steering system to track a yaw rate reference using a PI controller.

In this paper a similar tire force approximation is used for the design of a state-feedback PWA controller for a LKAS, which is able to operate in the entire tire force domain.

\footnotetext{
1 IFSTTAR - LIVIC, 14 Route de la Minière, 78000 Versailles, France (andre.benine-neto@ifsttar.fr)

3 IBISC - EA 4526 UEVE, 40 rue du Pelvoux CE1455, 91020, Evry, Cedex, France (e-mail: said.mammar@iup.univ-evry.fr)
}

Following the results obtained in [16], the control synthesis is casted as a Bilinear Matrix Inequality (BMI) optimization problem which is solved using the $\mathrm{V}-\mathrm{K}$ method. This interactive procedure allows the BMI to be transformed into two Linear Matrix Inequalities (LMI) optimizations problems which can be solved more efficiently to find simultaneously the state feedback gain and a Piecewise Quadratic Lyapunov (PWQL) function that ensure stability of the closed loop system. The vehicle model for control synthesis is described in Section II, followed by the controller design shown in Section III. The simulation results obtained on a nonlinear model are presented in Section IV providing a comparison of the PWA controller with a controller designed only for the linear behavior of the tire forces. Section $V$ wraps up the work and provides some perspective for the future work.

\section{VEHICLE MODEL}

In order to design the control law for the LKAS, a simple nonlinear vehicle model is used, in which the lateral translational and yaw motions are considered and the roll and pitch motions are neglected. The wheels of the front and rear axles are lumped into one located at the axle center leading to a bicycle model. The equations describing this model are given by:

$$
\left\{\begin{aligned}
m v(\dot{\beta}+r) & =f_{s f}+f_{s r} \\
J \dot{r} & =l_{f} f_{s f}-l_{r} f_{s r},
\end{aligned}\right.
$$

where the involved variables consist of the vehicle yaw rate, denoted $r$ and the vehicle sideslip angle, $\beta$. Concerning the fixed parameters for the model, $v$ represents the longitudinal vehicle speed, $m$ is the vehicle mass, $l_{f}\left(l_{r}\right)$ is the distance from the front (rear) axle to the center of gravity $(\mathrm{CG}), J$ is the vehicle inertia with respect to the vertical axle through the CG. All numerical values of these parameters are presented in Table I. The lateral forces $f_{s x}$, with $x=f, r$, for the front and rear tires can be modeled according to the Pacejka tire model [14]:

$$
f_{s x}\left(\alpha_{x}\right)=D_{x} \sin \left\{C_{x} \operatorname{atan}\left[\left(1-E_{x}\right) B_{x} \alpha_{x}+E_{x} \operatorname{atan}\left(B_{x} \alpha_{x}\right)\right]\right\},
$$

where $\alpha_{x}$ stands for the front (rear) tire sideslip angle. Considering that the angles remain small, the sideslip angles for front and rear tires are given by:

$$
\alpha_{f}=\delta_{f}-\beta-\frac{l_{f} r}{v}, \quad \alpha_{r}=-\beta+\frac{l_{r} r}{v},
$$

where $\delta_{f}$ is the steering angle.

The Pacjeka tire model is depicted by the solid line in Fig. 1 


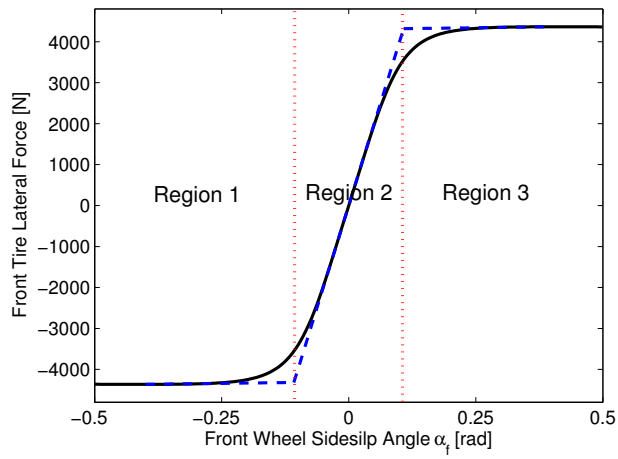

Fig. 1. Front tire forces described by the Pacejka magic formula and corresponding PWA approximations and partitions.

The simple nonlinear model (1) shows, as well known in literature (see for example [13]), a limited stability region which also depends on the driver steering wheel angle, two saddle points and a stable one (bifurcation analysis). The causes of the instability are due to the nonlinear behavior of the tire-road forces which are taken into account in the PWA vehicle model described in the next section.

\section{A. Piecewise affine approximation of lateral tire forces}

Considering that the front and rear tire forces described by (2) are approximated by the following PWA functions:

$$
\left\{\begin{array}{c}
f_{s f}\left(\alpha_{f}\right)=e_{f i}+d_{f i} \alpha_{f} \\
f_{s r}\left(\alpha_{r}\right)=e_{r i}+d_{r i} \alpha_{r}
\end{array}\right.
$$

where $d_{f i}, d_{r i}, e_{f i}$ and $e_{r i}$ depend on the front and rear tire forces and the index $(i)$ corresponding to the partitioning of the tire force domain, based on the tire sideslip angles, as illustrated by the dotted lines on Fig. 1 for the front wheel.

Replacing the PWA approximation of lateral tire force (4) in the vehicle model (1) leads to the PWA system:

$$
\dot{x}^{d}=A_{i}^{d} x^{d}+B_{i}^{d} u+a_{i}^{d},
$$

with the corresponding dynamics:

$$
\begin{gathered}
A_{i}^{d}=\left[\begin{array}{cc}
-\frac{d_{f i}+d_{r i}}{m v} & -1-\frac{d_{f i} l_{f}-d_{r i} l_{r}}{m v^{2}} \\
\frac{d_{r i} l_{r}-d_{f i} l_{f}}{J} & -\frac{d_{f i} l_{f}^{2}+d_{r i} l_{r}^{2}}{J v}
\end{array}\right], \\
B_{i}^{d}=\left[\begin{array}{c}
\frac{d_{f i}}{m v} \\
\frac{d_{f i} l_{f}}{J}
\end{array}\right], \quad a_{i}^{d}=\left[\begin{array}{c}
\frac{e_{f i}+e_{r i}}{m v} \\
\frac{e_{f i} l_{f}-e_{r i} l_{r}}{J}
\end{array}\right] .
\end{gathered}
$$

where the control input is the front wheel steering angle $u=\delta_{f}$ and the state variables are the vehicle sideslip angle, $\beta$ and the vehicle yaw rate, $r, x^{d}=[\beta, r]^{T}$.

Even though this approximation can be refined, it is shown in the simulation section that improved performance can be obtained by simply approximating the tire forces with the proposed PWA functions.
TABLE I

VALUES OF THE VEHICLE PARAMETERS.

\begin{tabular}{|l|l|}
\hline Parameter & Value \\
\hline$c_{f}$, front cornering stiffness & $40000 \mathrm{~N} / \mathrm{rad}$ \\
$c_{r}$, rear cornering stiffness & $35000 \mathrm{~N} / \mathrm{rad}$ \\
$l_{f}$, distance form CG to front axle & $1.22 \mathrm{~m}$ \\
$l_{r}$, distance form CG to rear axle & $1.44 \mathrm{~m}$ \\
$l_{s}$, look-ahead distance & $0.95 \mathrm{~m}$ \\
$m$, total mass & $1600 \mathrm{~kg}$ \\
$J$, vehicle yaw moment of inertia & $2454 \mathrm{kgm}^{2}$ \\
$v$, longitudinal velocity & $17 \mathrm{~m} / \mathrm{s}$ \\
\hline
\end{tabular}

\section{B. Additional dynamics for lane keeping}

For lane keeping purposes, the model (5) has to be expanded with the dynamics of the relative yaw angle and the lateral displacement with respect to the lane centerline. These measurements are provided by a video sensor. Let $\psi_{L}=\psi-\psi_{d}$ be the yaw angle error which is the angle between the vehicle orientation and the tangent to the road. The road reference curvature $\rho_{\text {ref }}$ is defined by $\left(\dot{\psi}_{d}=v \rho_{r e f}\right)$, and the following equality can be derived:

$$
\dot{\psi}_{L}=r-v \rho_{r e f}
$$

Denoting by $l_{s}$ the look-ahead distance, the equation giving the evolution of the measurement of the lateral offset $y_{L}$ from the centerline at sensor location is obtained by

$$
\dot{y}_{L}=v\left(\beta+\psi_{L}\right)-l_{s} r
$$

Equations (8) and (9) are included in system (5) and the PWA system describing the vehicle dynamics and positioning is described by:

$$
\dot{x}^{p}=A_{i}^{p} x^{p}+B_{i}^{p} u+B_{\rho}^{p} \rho_{r e f}+a_{i}^{p}
$$

where $x^{p}=\left[\beta, r, \psi_{L}, y_{L}\right]^{T}$ and

$$
\begin{aligned}
A_{i}^{p} & =\left[\begin{array}{cccc}
A_{i}^{d} & 0 & 0 \\
0 & 1 & 0 & 0 \\
v & l_{s} & v & 0
\end{array}\right], \\
B_{i}^{p} & =\left[\begin{array}{lll}
\left(B_{i}^{d}\right)^{T} & 0 & 0
\end{array}\right]^{T}, \\
B_{\rho}^{p} & =\left[\begin{array}{cccc}
0 & 0 & -v & 0
\end{array}\right]^{T}, \\
a_{i}^{p} & =\left[\begin{array}{lll}
\left(a_{i}^{d}\right)^{T} & 0 & 0
\end{array}\right]^{T} .
\end{aligned}
$$

An illustration of the state variables is provided in Fig. 2

\section{Control Strategy}

The algorithm presented in [16] is applied for the design of the proposed LKAS. It provides an efficient procedure for control synthesis for PWA systems using a PWA state feedback gain, by means of a BMI optimization procedure, based on the search of a PWQL function. 


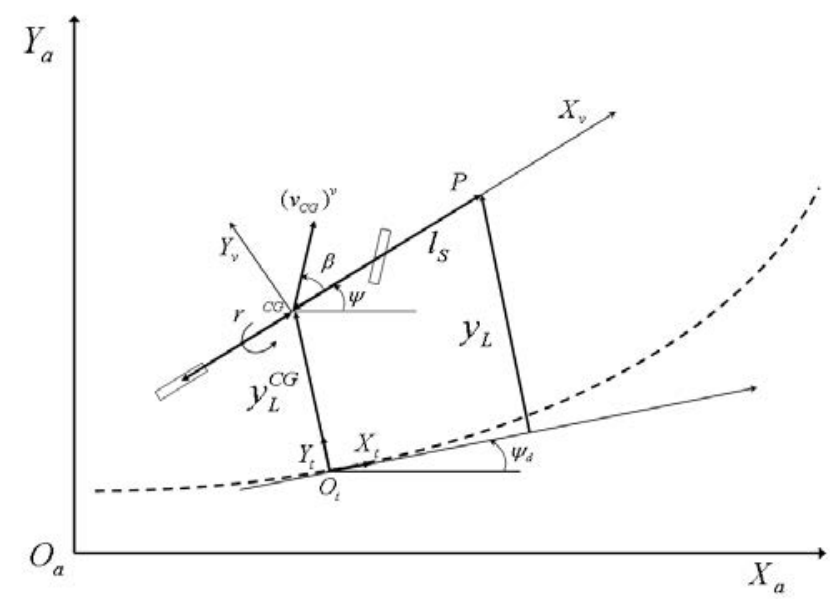

Fig. 2. Single track vehicle model.

\section{A. Assumptions for LKAS and PWA system description}

As most vehicles have understeering behavior, it is assumed that the front tire forces tend to saturate firstly, therefore only the front tire forces are approximated by PWA functions according to (4), and a single linear approximation is assumed for the rear tire, as follows:

$$
\begin{aligned}
& \begin{cases}f_{s f}\left(\alpha_{f}\right)=e_{f 1}+d_{f 1} \alpha_{f}, & \text { for } \alpha_{f}<-\bar{\alpha}_{f}, \\
f_{s f}\left(\alpha_{f}\right)=c_{f} \alpha_{f}, & \text { for }-\bar{\alpha}_{f}<\alpha_{f}<\bar{\alpha}_{f}, \\
f_{s f}\left(\alpha_{f}\right)=e_{f 3}+d_{f 3} \alpha_{f}, & \text { for } \alpha_{f}>\bar{\alpha}_{f},\end{cases} \\
& \left\{\begin{array}{l}
f_{s r}\left(\alpha_{r}\right)=c_{r} \alpha_{r} \quad \forall \alpha_{r},
\end{array}\right.
\end{aligned}
$$

where $c f=B_{f} C_{f} D_{f}, c_{r}=B_{r} C_{r} D_{r}$ and $\bar{\alpha}_{f}$ corresponds to the partition shown in Fig. 1.

According to (3), the partitions defined by the front wheel sideslip angle, $\alpha_{f}= \pm \bar{\alpha}_{f}$, depend on the state variables and control input. As mentioned in [16] the switching between the regions should depend only on the state $x$, so models in which there is a feed-through from $u$ to a nonlinearity should be avoided. This can be done by the inclusion of a first order actuator which is also useful to enforce continuity of the control input at the boundaries. Therefore, the steering angle is included as an additional state to the system as follows:

$$
\dot{\delta}_{f}=-\tau \delta_{f}+u_{c}
$$

where $u_{c}$ becomes the control input of the augmented plant and $\tau=10$.

Disturbances and exogenous inputs, such as the road curvature, are not taken into account in the control synthesis, therefore the model describing the vehicle dynamics, positioning (10) and first order actuator (13) can be written as:

$$
\dot{x}=A_{i} x+B_{i} u+a_{i},
$$

with $x=\left[\beta, r, \psi_{L}, y_{L}, \delta_{f}\right]^{T}$ and

$$
\begin{aligned}
A_{i} & =\left[\begin{array}{ccccc} 
& A_{i}^{d} & & B_{i}^{p} \\
0 & 0 & 0 & 0 & -\tau
\end{array}\right], \\
B_{i} & =\left[\begin{array}{lllll}
0 & 0 & 0 & 0 & \tau
\end{array}\right]^{T}, \\
a_{i} & =\left[\begin{array}{ll}
\left(a_{i}^{d}\right)^{T} & 0
\end{array}\right]^{T} .
\end{aligned}
$$

Each of the regions $R_{i}, i=1,2,3$ can be described as the intersection of the half spaces

$$
R_{i}=\left\{x \mid H_{i}^{T} x-g_{i}<0\right\}
$$

Since these regions are slab, it is preferred to express them as degenerated ellipsoids, as suggested in [15] and [16], because they describe exactly the slab regions and it often requires fewer parameters than the polytopic description (16).

The degenerated ellipsoids can be described by:

$$
\left\|E_{i} x+f_{i}\right\|_{2} \leq 1 \quad \forall x \in R_{i},
$$

and following the description in [15], if $R_{i}=\left\{x \mid \underline{\mathrm{d}}<c_{i}^{T} x<\bar{d}\right\}$ then the degenerated ellipsoid is described by:

$$
E_{i}=2 c_{i}^{T} /(\bar{d}-\underline{\mathrm{d}}) \quad \text { and } \quad f_{i}=-(\bar{d}+\underline{\mathrm{d}}) /(\bar{d}-\underline{\mathrm{d}})
$$

For the PWA system (14), the ellipsoids describing the slab regions are:

$$
\begin{array}{ll}
E_{1}^{T}=\left[-1,-\frac{l_{f}}{v}, 0,0,1\right] \frac{2}{-\bar{\alpha}_{f}-\underline{\mathrm{d}}}, & f_{1}=-\frac{-\bar{\alpha}_{f}+\underline{\mathrm{d}}}{-\bar{\alpha}_{f}-\underline{\mathrm{d}}}, \\
E_{2}^{T}=\left[-1,-\frac{l_{f}}{v}, 0,0,1\right] \frac{1}{\bar{\alpha}_{f}}, & f_{2}=-\frac{\bar{\alpha}_{f}-\bar{\alpha}_{f}}{\bar{\alpha}_{f}+\bar{\alpha}_{f}}=0, \\
E_{3}^{T}=\left[-1,-\frac{l_{f}}{v}, 0,0,1\right] \frac{2}{\bar{d}-\bar{\alpha}_{f}}, & f_{3}=-\frac{\bar{d}+\bar{\alpha}_{f}}{\bar{d}-\bar{\alpha}_{f}},
\end{array}
$$

where $\underline{\mathrm{d}}$ and $\bar{d}$ can be arbitrarily set, such that $\underline{\mathrm{d}}<-\bar{\alpha}_{f}$ and $\bar{d}>\bar{\alpha}_{f}$.

The ellipsoidal cell description is used to relax the constraints when searching the PWQL function. In order to ensure the continuity of the PWQL function across the boundaries, a parametric boundary description is needed [8]

Each facet boundary between the neighboring regions $R_{i}$ and $R_{j}$ is contained in the hyperplanes described by:

$$
\left\{x \mid c_{i j}^{T} x-d_{i j}=0\right\} .
$$

and the boundary parametric description can be obtained by:

$$
\bar{R}_{i} \cap \bar{R}_{j} \subseteq\left\{x / l_{i j}+F_{i j} s / s \in R^{n-1}\right\}
$$

where $F_{i j} \in R^{n \times n-1}$ (full rank) is the matrix whose columns span the null space of $c_{i j}$, and $l_{i j} \in R^{n}$ is given by $l_{i j}=$ $c_{i j}\left(c_{i j}^{T} c_{i j}\right)^{-1} d_{i j}$

For the LKAS model (14) the parametric description of the boundaries is given by:

$$
F_{1,2}=\left[\begin{array}{cccc}
1 & 0 & 0 & 0 \\
0 & 1 & 0 & 0 \\
0 & 0 & 1 & 0 \\
0 & 0 & 0 & 1 \\
1 & \frac{l_{f}}{v} & 0 & 0
\end{array}\right], F_{2,3}=\left[\begin{array}{cccc}
1 & 0 & 0 & -\frac{l_{f}}{v} \\
0 & 0 & 0 & 1 \\
0 & 1 & & 0 \\
0 & 0 & 1 & 0 \\
1 & 0 & 0 & 0
\end{array}\right]
$$




$$
\begin{aligned}
& l_{1,2}=\left[\begin{array}{lllll}
\frac{\bar{\alpha}_{f}}{2+l_{f}^{2} / v^{2}}, & \frac{\bar{\alpha}_{f} l_{f} / v}{2+l_{f}^{2} / v^{2}}, & 0, & 0, & -\frac{\bar{\alpha}_{f}}{2+l_{f}^{2} / v^{2}}
\end{array}\right], \\
& l_{2,3}=\left[\begin{array}{lllll}
-\frac{\bar{\alpha}_{f}}{2+l_{f}^{2} / v^{2}}, & -\frac{\bar{\alpha}_{f} l_{f} / v}{2+l_{f}^{2} / v^{2}}, & 0, & 0, & \frac{\bar{\alpha}_{f}}{2+l_{f}^{2} / v^{2}}
\end{array}\right] .
\end{aligned}
$$

\section{B. PWA State-Feedback control synthesis}

Due to the symmetry of the lateral tire force with respect to the origin, only regions $R_{1}$ and $R_{2}$ are considered in the control synthesis. The same gains obtained for region $R_{1}$ can be applied in region $R_{3}$.

The goal is to stabilize (14) with a PWA state feedback gain $u_{c}=K_{i} x+m_{i}$, based on the search of a PWQL function of the form:

$$
V_{i}(x)=x^{T} P_{i} x+2 q_{i}^{T} x+r_{i}
$$

$V_{i}(x)$ can be a Lyapunov function with a decay rate $\alpha_{i}$, for the region $R_{i}$ if, for a fixed $\varepsilon \geq 0$,

$$
x \in R_{i},\left\{\begin{array}{c}
V_{i}(x)>\varepsilon\left\|x-x_{e q}\right\|_{2} \\
\frac{d}{d t} V_{i}(x)<-\alpha_{i} V_{i}(x),
\end{array}\right.
$$

where $x_{e q}$ is the equilibrium point of the closed loop system.

Rendering the vehicle to the center of the lane is a regulation problem for system (14), since in a straight line all state variables should converge to the origin. Therefore the desired equilibrium point of the closed loop system, $x_{e q}=0$, is placed at the origin. The equilibrium point must also be the extrema of the Lyapunov function candidate for the corresponding region (see [16]). In consequence, $q_{2}=0_{5 \times 1}$ and $r_{2}=0$ to ensure that $V(0)=0$.

The closed-loop state-space equation is:

$$
\dot{x}=\left(A_{i}+B_{i} K_{i}\right) x+\left(b_{i}+B_{i} m_{i}\right)=\tilde{A}_{i} x+\tilde{b}_{i} .
$$

As the closed loop system has a single equilibrium point, the equilibrium points of $R_{1}$, denoted $x_{e q 1}$ and $R_{3}, x_{e q 3}$, must not be contained in its own region, enforcing a transition through the boundaries with $R_{2}$. This can be done using the constraint:

$$
\left(A_{i}+B_{i} K_{i}\right) x_{e q}+\left(b_{i}+B_{i} m_{i}\right)=0 .
$$

The stability of the (25) can be guaranteed by S-procedure [5], using the ellipsoidal cell description (17) and conditions (24), if there exists $P_{i}=P_{i}^{T} \succ 0$, and positive constants $\lambda_{1}$ and $\gamma_{1}$ that satisfy:

$$
\begin{aligned}
& \left\{\begin{array}{cc}
{\left[\begin{array}{cc}
P_{1}-\varepsilon I_{n}+\lambda_{1} E_{1}^{T} E_{1} & q_{1}+\varepsilon x_{e q}+\lambda_{1} E_{1}^{T} f_{1} \\
* & r_{1}-\varepsilon x_{1}^{T} x_{1}+\lambda_{1}\left(f_{1}^{T} f_{1}-1\right)
\end{array}\right] \succ 0} \\
{\left[\begin{array}{c}
\left.P_{2}-\varepsilon I_{n}\right] \succ 0 \\
*
\end{array}\right.} \\
\left\{\begin{array}{c}
{\left[\begin{array}{c}
P_{1} \tilde{b}_{1}+\tilde{A}_{1}^{T} q_{1} \\
-\gamma_{1} E_{1}^{T} f_{1}+\alpha_{1} q_{1}
\end{array}\right)} \\
-\gamma_{1} E_{1}^{T} E_{1}+P_{1} P_{1}
\end{array}\right) \\
\left.\begin{array}{c}
2 \tilde{b}_{1}^{T} q_{1}+\alpha_{1} r_{1} \\
-\gamma_{1}\left(f_{1}^{T} f_{1}-1\right)
\end{array}\right)
\end{array}\right] \prec 0
\end{aligned}
$$

Using the parametric description of the boundaries (22), the continuity of the PWQL function candidate is ensured by:

$$
\left\{\begin{aligned}
F_{12}^{T}\left(P_{1}-P_{2}\right) F_{12} & =0 \\
F_{12}^{T}\left(P_{1}-P_{2}\right) l_{12}+F_{12}^{T} q_{1} & =0 \\
l_{12}^{T}\left(P_{1}-P_{2}\right) l_{12}+2 q_{1}^{T} l_{12}+r_{1} & =0
\end{aligned}\right.
$$

Ensuring that there is no sliding mode between $R_{1}$ and $R_{2}$ can be enforced by the equations:

$$
\left\{\begin{array}{r}
c_{12}^{T}\left(\tilde{A}_{1}-\tilde{A}_{2}\right) F_{12}=0 \\
c_{12}^{T}\left(\tilde{A}_{1}-\tilde{A}_{2}\right) l_{12}+\tilde{b}_{1}-\tilde{b}_{2}=0
\end{array}\right.
$$

Defining a performance criterion as: $J=\min _{i} \alpha_{i}$. The goal is to find the PWA state feedback controller that maximizes the performance criterion $J$. This optimization problem can be cast as a BMI as:

maximise: $\quad \min _{i} \alpha_{i}$

subjected to: (26), (29), (27), (28), (30),

$$
\begin{aligned}
& \varepsilon>0, \quad \gamma_{1}>0, \quad \lambda_{1}>0, \quad \alpha_{1,2}>l_{0}>0, \\
& -l_{1}<K_{i}<l_{1}, \quad-l_{2}<m_{i}<l_{2}
\end{aligned}
$$

where $l_{0}$ is a scalar bound, $l_{1}$ and $l_{2}$ are vector bounds.

The BMI problem (31) can be solved using the V-K method, in which LMIs are solved in an iterative fashion. For that, the equilibrium point of the dynamics corresponding to the affine region $x_{e q 1}$, is chosen a-priori, such that it is not located in $R_{1}$. For this specific lane keeping problem, the algorithm becomes.

$V$-step: Given a fixed controller, and a fixed $\alpha_{i}$, solve:

Find: $\quad P_{1}, q_{1}, r_{1}$ and $P_{2}$,

s. t. : (29), (27), (28),

$$
\varepsilon>0, \quad \gamma_{1}>0, \quad \lambda_{1}>0
$$

K-step: For $P_{1}, q_{1}, r_{1}$ and $P_{2}$ fixed at the previous step, solve:

$$
\begin{array}{ll}
\operatorname{maximise} & \min _{i} \alpha_{i} \\
\text { s. t.: } & (26),(27),(28),(30), \\
& \varepsilon>0, \quad \gamma_{1}>0, \quad \lambda_{1}>0, \quad \alpha_{1,2}>l_{0}>0, \\
& -l_{1}<K_{i}<l_{1}, \quad-l_{2}<m_{i}<l_{2},
\end{array}
$$

For each iteration of the K-step, the decay rates $\alpha_{1}$ and $\alpha_{2}$ must be greater than the value computed at the previous iteration. The loop must be repeated until there is no significant improvement on the cost or the LMIs become infeasible.

The gain for the first iteration on the V-step is computed using the algorithm from [12]. The same gain is considered for both regions, and $m_{1}=m_{2}=0$. 


\section{Simulation Results}

The resulting gains from the V-K method are:

$$
\begin{gathered}
K_{1}=[-0.0914-0.1514-1.0289-0.0824-0.1919], \\
K_{2}=[-0.3184-0.1639-1.0289-0.0824-0.1879], \\
K_{3}=K_{1}, \quad m_{1}=-m_{3}=0.0245, m_{2}=0
\end{gathered}
$$

In order to verify the performance of the PWA controller, simulation were carried out on the Matlab/Simulink environment, comparing two vehicles, one equipped with the PWA controller and another equipped with the controller computed for the linear region only (controller $K_{2}$ ). The dynamics of the model used for simulation consist of nonlinear 4-wheels vehicle according to [1], which is considered to capture the essential vehicle lateral steering dynamics:

$$
\begin{aligned}
m\left(\dot{v}_{y}+r v\right) & =f_{s f} \cos \delta_{f}+f_{s r} \\
J \dot{r} & =l_{f} f_{s f} \cos \delta_{f}-l_{r} f_{s r}
\end{aligned}
$$

where $v_{y}$ is the lateral velocity, and the lateral forces are given by (2). The sideslip angles $\alpha_{f, r}$ are given by:

$$
\begin{aligned}
& \alpha_{f(l e f t)}=\delta_{f}-\arctan \left(\frac{v \beta+r l_{f}}{v-\frac{a}{2} r}\right) \\
& \alpha_{f(r i g h t)}=\delta_{f}-\arctan \left(\frac{v \beta+r l_{f}}{v+\frac{a}{2} r}\right) \\
& \alpha_{r(l e f t)}=-\arctan \left(\frac{v \beta-r l_{r}}{v-\frac{a}{2} r}\right) \\
& \alpha_{r(r i g h t)}=-\arctan \left(\frac{v \beta-r l_{r}}{v+\frac{a}{2} r}\right)
\end{aligned}
$$

As an attempt to make the lane departure avoidance assistance system unobtrusive during normal driving, the activation strategy takes place only in case the driver is inattentive and there is a risk of lane departure. Throughout the simulations the driver is considered inattentive, and analogously to [12] the risk of lane departure is estimated by position of the front wheels with respect to a centered strip on the lane with width $2 d$. The position of the left $\left(y_{l}\right)$ and right $\left(y_{r}\right)$ front wheels can be described by:

$$
\begin{aligned}
& y_{l}=y_{L}+\left(l_{f}-l_{s}\right) \psi_{L}+\frac{a}{2}, \\
& y_{r}=y_{L}+\left(l_{f}-l_{s}\right) \psi_{L}-\frac{a}{2},
\end{aligned}
$$

where $a$ represents the vehicle width. Enforcing that the front wheels must remain inside the centred lane strip $\pm d$, yields:

$$
-\frac{2 d-a}{2} \leq y_{L}+\left(l_{f}-l_{s}\right) \psi_{L} \leq \frac{2 d-a}{2}
$$

This set, corresponding to the region between two parallel hyperplanes, can be written with respect to the state vector as:

$$
T \triangleq\left\{x \in R^{5}:|F x| \leq 1\right\}
$$

where $F=\left(0,0, \frac{2\left(l_{f}-l_{s}\right)}{2 d-a}, \frac{2}{2 d-a}, 0\right)$.

The road adhesion is considered degraded, in order to show the performance of the controller in the occurrence of tire forces saturation. The road adhesion coefficient is considered $\mu=0.5$, which corresponds to wet pavement. This condition is incorporated in the parameters of (2) by changing $B$ to $(2-\mu) B, C$ to $\left(\frac{5}{4}-\frac{\mu}{4}\right) C$ and $D$ to $\mu D$, [10].

The simulation scenario consists of an inattentive driver approaching a left-hand side curve. As the right front wheel reaches the centered strip $d$ the LKAS system is activated.
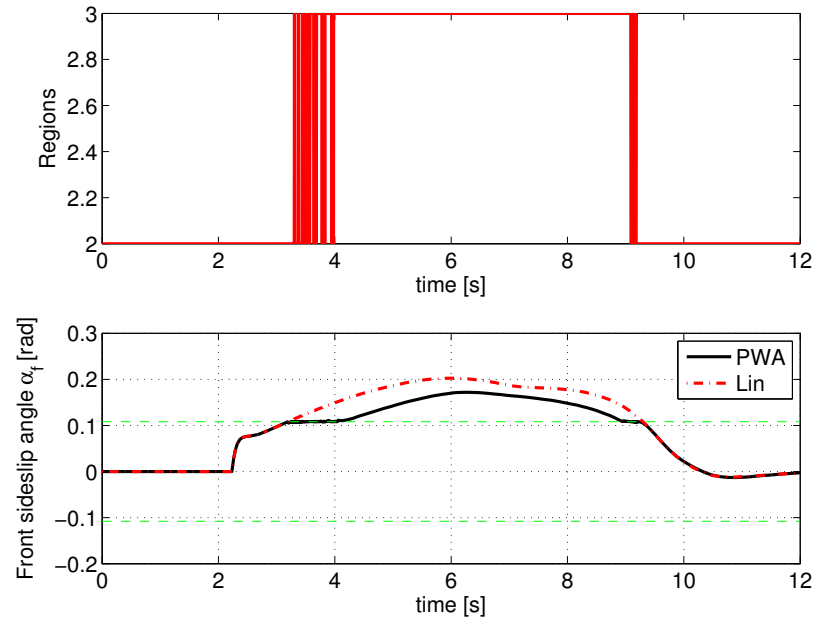

Fig. 3. Active region for PWA control and response for vehicles with PWA and linear controllers
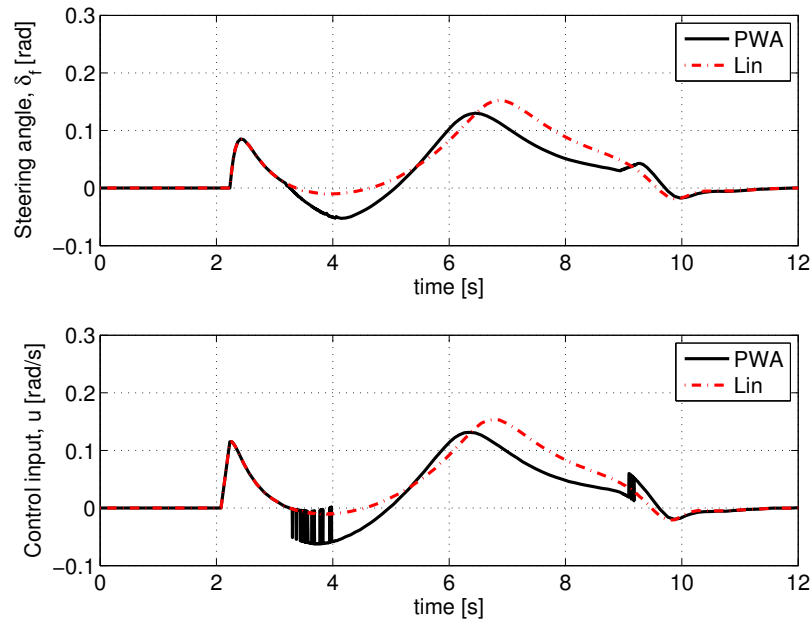

Fig. 4. Steering angle and Control input for vehicles with PWA and linear controllers

While attempting to steer the vehicle to the center of the lane, the front wheel sideslip angle, depicted in Fig. 3 overshoots the limit considered for the linear behavior, corresponding to the saturation of tire forces. The switches between the gains corresponding to $R_{2}$ and $R_{3}$ are shown in Fig. 3. The corresponding control input and steering angle that can be seen in Fig. 4.

The vehicle sideslip angle and yaw rate for the two vehicles are shown in subplots of Fig. 5. The relative yaw angle and lateral offset at look-ahead distance, representing the vehicle positioning on the road are depicted in Fig 6. It is interesting to note that the dynamics of the vehicle equipped with the PWA controller are less extreme due to the switching of the controller. This control action to reestablish vehicle stability leads to a slightly larger lateral offset during the maneuver, but it is still within an acceptable range. Moreover it avoids the saturation of the lateral tire forces. 

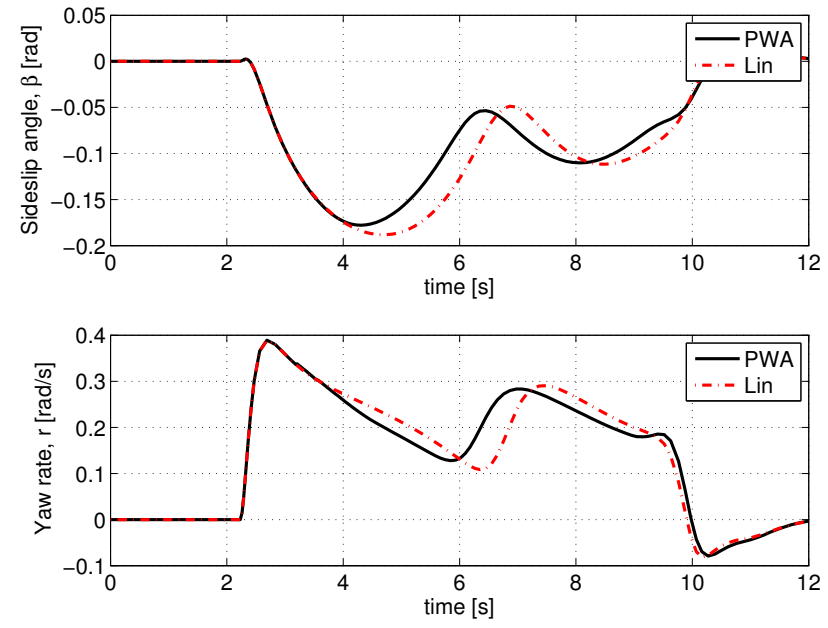

Fig. 5. Vehicle dynamics response for vehicles with PWA and linear controllers
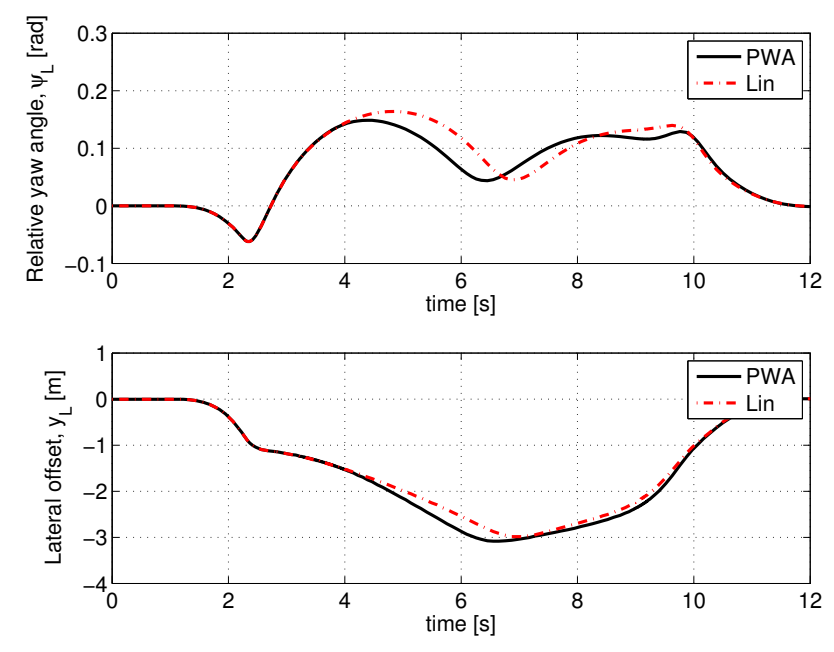

Fig. 6. Positioning for vehicles with PWA and linear controllers

\section{CONCLUSION}

In this paper the design and simulation of a PWA controller for LKAS has been described. The nonlinear behavior of the lateral tire forces are approximated by PWA functions. As only understeering vehicles are considered in this work, the regions describing the PWA vehicle model can be exactly described as degenerated ellipsiods which are used to relax the conditions for the existence of a PWQL function. The simultaneous search of a PWQL function and a PWA state feedback gain has been casted as a BMI optimization problem. The solution has been computed using the V$\mathrm{K}$ method in which the BMIs are transformed into two LMI optimization procedures that are solved iteratively. The resulting PWA controller has been tested in degenerated adherence conditions showing its enhanced performance under the effects of tire force saturation and the activation strategy of the assistance system.

Currently commercialized vehicles are not equipped with sensors that are able to measure the vehicle sideslip angles.
Therefore the design of a output feedback controller should be considered in the future work. Taking into account parameter variations as for example vehicle longitudinal speed, and implementation on prototype vehicle should also be included in the future work.

\section{ACKNOWLEDGMENT}

This work has been partially financed by the FUI French Initiative E-Motive.

\section{REFERENCES}

[1] J. Ackermann. Robust Control. Springer, London, 2002.

[2] A. Benine-Neto, S. Scalzi, M. Netto, S. Mammar, and W. PasillasLepine. Vehicle yaw rate control based on piecewise affine regions. In Intelligent Vehicles Symposium (IV), 2010 IEEE, San Diego, USA, pages 20-25, June 2010.

[3] D. Bernardini, S. D. Cairano, A. Bemporad, and H. Tseng. Driveby-wire vehicle stabilization and yaw regulation: A hybrid model predictive control design. In Joint 48th IEEE Conference on Decision and Control and 28th Chinese Control Conference, Shanghai, P.R. China, pages 7621-7626, December 2009.

[4] F. Borrelli, A. Bemporad, M. Fodor, and D. Hrovat. A Hybrid Approach to Traction Control. Springer Berlin / Heidelberg pp. 162174, 2001.

[5] S. Boyd, L. E. Ghaoui, E. Feron, and V. Balakrishnan. Linear Matrix Inequalties in System and Control Theory, chapter 7. SIAM, 1994.

[6] P. Falcone, F. Borrelli, J. Asgari, H. E. Tseng, and D. Hrovat. Predictive active steering control for autonomous vehicle systems. IEEE Transactions on control Systems Technology, 15(3):566-580, May 2007.

[7] R. Fenton, G. Melocik, and K. Olson. On the steering of automated vehicles : Theory and experiment. IEEE Transactions on on Automatic Control, 21:306-315, 1976.

[8] A. Hassibi and S. Boyd. Quadratic stabilization and control of piecewise-linear systems. In Proceedings of the American Control Conference, Philadelphia, PA, pages 3659-3664, 1998.

[9] C. Hatipoglu, Ü. Özgüner, and K. A. Redmill. Automated lane change controller design. IEEE Transactions on Intelligent Transportation Systems, 3(1):13-22, 2003.

[10] S. Mammar and D. Koenig. Vehicle handling improvement by active steering. Vehicle System Dynamics, 38(3):211-242, 2002.

[11] S. Mammar, N. Minoiu-Enache, S. Glaser, B. Lusetti, and A. BenineNeto. Lane keeping automation at tire saturation. In Proceeding of the 2010 American Control Conference, Baltimore, June 2010.

[12] N. Minoiu-Enache, M. Netto, S. Mammar, and B. Lusetti. Driver steering assistance for lane departure avoidance. Control Engineering Practice, 17:642-651, 2009.

[13] E. Ono, S. Hosoe, H. D. Tuan, and S. Doi. Bifurcation in vehicle dynamics and robust front wheel steering control. IEEE Trans. on Control System Technology, 6(3):9-14, May 1998.

[14] H. Pacejka. Tire and Vehicle Dynamics. Elsevier ButterworthHeinemann, 2004.

[15] L. Rodrigues and S. Boyd. Piecewise-affine state feedback for piecewise-affine slab systems using convex optimization. Systems and Control Letters, 54:835-853, 2005.

[16] L. Rodrigues and J. P. How. Observer based control of piecewise-affine systems. Int. J. Control, 76(5):459-477, 2003.

[17] S. Scalzi, A. Benine-Neto, M. Netto, W. Pasillas-Lepine, and S. Mammar. Active steering control based on piecewise affine regions. In Proceeding of the 2010 American Control Conference, Baltimore, June 2010.

[18] S. E. Shladover. Path at 20 - history and major milestones. IEEE Transactions on Intelligent Transportation Systems, 8(4):584-592, December 2007.

[19] E. D. Sontag. Nonlinear regulation: The piecewise linear approach IEEE Transactions on Automatic Control, AC-26(2):346-358, April 1981. 